\title{
Full radiative coupling in two-phase models for accreting black holes
}

\author{
J. Malzac ${ }^{1,2}$, A. M. Dumont ${ }^{3}$, and M. Mouchet ${ }^{3}$ \\ ${ }^{1}$ Centre d'Étude Spatiale des Rayonnements, CNRS-UPS, 9 avenue du Colonel Roche, 31028 Toulouse Cedex 4, France \\ e-mail:malzac@cesr.fr \\ 2 Institute of Astronomy, Madingley Road, Cambridge, CB3 OHA, UK \\ ${ }^{3}$ LUTH, UMR 8102 (CNRS/Université Paris 7), Observatoire de Paris, Section de Meudon, 92195 Meudon Cedex, France
}

Received 21 January 2003 / Accepted 21 July 2004

\begin{abstract}
The emission from galactic black holes and Seyfert galaxies is generally understood in term of two-phase models (Haardt \& Maraschi 1991, 1993). Such models postulate that a hot plasma $\left(\sim 10^{9} \mathrm{~K}\right)$ coexists with relatively colder material $\left(\sim 10^{6} \mathrm{~K}\right)$ in the inner part of the accretion flow. We present the first simulated broad-band spectra produced by such a system and accounting simultaneously for energy balance and Comptonisation in the hot phase, together with reflection, reprocessing, ionization and thermal balance in the cold phase. This was made possible by coupling three radiative transfer codes: a nonlinear Monte-Carlo code (NLMC), a photo-ionization code TITAN and a linear Monte-Carlo code NOAR. The equilibrium comptonisation spectrum appears to be sensitive to the shape of the reprocessed spectrum that, in turn, depends on the ionization parameter, but also on the structure of the irradiated cold material. This is illustrated by a comparison of simulations assuming constant density or a constant pressure in the cold phase. We also compare our results with simplified models where reprocessing is approximated by a blackbody spectrum. Our detailed treatment leads to noticeably different spectral energy distributions (SEDs) characterised by harder X-ray spectra. Even at low ionization parameters $\left(\xi \sim 300 \mathrm{erg} \mathrm{s}^{-1} \mathrm{~cm}\right)$ the commonly used blackbody approximation is poor, leading to X-ray spectra that are too soft. The effect, however, seems not to be strong enough to reconcile the slab corona model with the hardest observed spectra, unless the reflector has a constant density and the ionization parameter is large, of the order of $10^{4} \mathrm{erg} \mathrm{s}^{-1} \mathrm{~cm}$.
\end{abstract}

Key words. accretion, accretion disks - black hole physics - radiative transfer - method: numerical - galaxies: Seyfert $\mathrm{X}$-ray: binaries

\section{Introduction}

At least two distinct mediums are required to produce the main features observed in the broad band spectra of radio quiet active galactic nuclei (AGN) and black hole binaries (BHBs) in the low/hard state. Their hard X-ray emission exhibits a powerlaw spectrum cutting-off at a few hundred $\mathrm{keV}$, which is generally interpreted as thermal Comptonisation spectra in a very hot $\left(\sim 10^{9} \mathrm{~K}\right)$ optically thin plasma with Thomson depth $\tau_{\mathrm{T}} \sim 1$ (Sunyaev \& Titarchuk 1980; Haardt \& Maraschi 1991; Stern et al. 1995b; Poutanen \& Svensson 1996; Zdziarski et al. 1998). The big blue bump observed in the UV spectra of Seyfert galaxies (Walter \& Fink 1993), as well as the soft X-ray excess in BHBs (e.g. Balucinska-Church et al. 1995) are indicative of thermal emission from optically thick and much colder material with a temperature in the range $10^{5}-10^{7} \mathrm{~K}$. The presence of relatively cold material in the innermost part of the accretion flow is corroborated by the existence of reflection features in the X-rays such as the Fe fluorescence line around $6.4 \mathrm{keV}$ and a reflection bump peaking at about $30 \mathrm{keV}$ (George \& Fabian 1991; Nandra \& Pounds 1994).
The nature and geometry of these two phases are uncertain. The cold medium is generally believed to form an accretion disc (Shakura \& Sunyaev 1973), alternatively it could consist of cold dense clouds located inside or around the hot plasma (Rees 1987; Collin-Souffrin et al. 1996; Malzac 2001; Malzac \& Celotti 2002). The hot phase could constitute the accretion disc corona (Svensson \& Zdziarski 1994; Życki et al. 1995; Sincell \& Krolik 1997; Różańska \& Czerny 2000, and Ref. therein) or the hot inner part of the accretion disc itself (Shapiro \& Lightman 1976).

It was soon realized that, if close to each other, the hot and the cold phases should be strongly radiatively coupled. This led to the development of two-phase models (Haardt \& Maraschi 1991, 1993), where the cold phase constitutes the main source of seed photons for the Comptonisation process. The temperature in the hot phase, and thus the detailed shape of the high energy spectrum, is controlled mainly by the flux of soft cooling photons. On the other hand, the thermal reprocessing of the high energy radiation impinging on the cold phase provides a dominant contribution to soft emission from the cold phase. 
Due to the complexity of the Comptonisation process, the importance of geometric effects, and the requirement of taking accurately into account the coupling between the two phases, it was necessary to develop sophisticated numerical codes in order to compute the detailed spectrum produced by the twophase system in different configurations (Stern et al. 1995a; Poutanen \& Svensson 1996).

The methods developed in this context, such as the nonlinear Monte-Carlo method (Stern et al. 1995a), provide an accurate treatment of the hot phase emission in energy balance. On the other hand, the emission from the cold phase is not detailed: a pure blackbody spectrum with a fixed temperature and reflection on neutral material are generally assumed.

Actually the emission due to X-ray reprocessing in the cold phase differs widely from a simple blackbody, in particular there is a complex line emission. Moreover, due to the hard X-ray irradiation, a ionised skin is likely to form on the surface layers of the cold material (Ross \& Fabian 1993; Collin-Souffrin et al. 1996; Nayakshin et al. 2000; Ballantyne et al. 2001). This ionised material affects the line emission as well as the shape and amplitude of the reflection component (Życki et al. 1994; Dumont et al. 2000; Nayakshin et al. 2000; Nayakshin \& Kallman 2001). Ionization is also likely to affect the equilibrium in the hot phase. Indeed the hard X-ray albedo for ionised material is larger and, as a consequence, the flux of thermal reprocessed soft photons is lower, affecting the temperature in the hot plasma.

The first attempts to include these effects in the two-phase model calculations used a very simplified approach to the computation of the reprocessed spectrum (e.g. Nayakshin \& Dove 2001). Indeed, a detailed self-consistent computation of the emission from irradiated material is an heavy task. It requires one to solve the radiative transfer equations in the cold medium taking into account the energy and ionization balance including all the atomic processes. Another difficulty comes from the fact that the cold material is optically thick and its temperature and ionization structure at equilibrium is strongly inhomogeneous.

The detailed computation of the structure and spectrum of irradiated optically thick material was performed by coupling the photo-ionization code TITAN and the Monte-Carlo code NOAR (accounting for Compton scattering) (Dumont et al. 2000). It enabled one to show the importance of an accurate radiative transfer treatment for the irradiated cold material emission.

However, in these calculations the feedback from the cold radiation on the hot phase equilibrium was not considered. As irradiating spectrum, a simple power-law with a fixed slope was assumed.

Here we add a self-consistent treatment of the hot phase emission by coupling the TITAN/NOAR codes with a third code based on the Non-Linear Monte-Carlo method (Malzac \& Jourdain 2000).

This enables us to propose the first full treatment of the radiative coupling, accounting accurately and self-consistently for both the hot and cold phase emission.

\section{The numerical model}

\subsection{The NOAR/TITAN and NLMC codes}

TITAN is a code designed for warm media $\left(T>\right.$ a few $\left.10^{4} \mathrm{~K}\right)$ optically thick to Compton scattering. It computes the structure of a plane-parallel slab of gas in thermal and ionization equilibrium, illuminated by a given spectrum on one or two sides of the slab (Dumont et al. 2000). It takes into account the returning flux using a two-stream approximation to solve the transfer in the lines (instead of the escape probability formalism). This code is coupled with a Monte Carlo code, NOAR, which takes into account Compton and inverse Compton diffusions in any geometry (Abrassart 2000). NOAR uses the local fractional ion abundances and the temperature provided by TITAN, while NOAR provides TITAN with the local Compton gains and losses in each layer. The Compton heating-cooling rate is indeed dominated by energy losses of photons at high energies $(>25 \mathrm{keV})$, not considered by TITAN. The coupling thus allows one to solve consistently both the global and the local energy balance. NOAR also allows computing the fluorescence line profiles which are significantly Compton-broadened in the case of strong illumination, and the Comptonised reflection spectrum above $25 \mathrm{keV}$.

In parallel, we use the NLMC code described and tested in Malzac \& Jourdain (2000) based on the Non-Linear MonteCarlo method proposed by Stern et al. (1995a). This code computes the Comptonised spectrum and energy balance in the hot phase. The radiation field is represented using about $10^{4}$ pseudo-particles called Large Particles (LP). Each LP represents a number of photons with identical characteristics (energy, position, direction of propagation, ...). These LPs are tracked all together in a synchronized way. They may interact by the Compton effect with a Maxwellian electron distribution. These interactions are simulated using standard Monte-Carlo methods. For a fixed heating rate in the hot phase, the temperature of the electron distribution is modified according to the Compton energy losses due to the interactions with the LP photons. Starting from an arbitrary radiation field and temperature the system evolves naturally toward equilibrium. Then, the escaping LP characteristics are used to build up the angle dependent spectrum from the hot phase until satisfying photon statistics are achieved.

\subsection{Geometry and model parameters}

The proposed method can apply to a variety of geometries of the two-phase system. However as the coupled TITAN/ NOAR/NLMC simulations are very time-consuming, we will limit this first attempt of full radiative coupling to simple situations. We will consider both the slab and spherical geometry illustrated in Fig. 1. The slab geometry, used e.g. by Malzac \& Jourdain (2000), corresponds to the standard infinite slab accretion disc corona model (Haardt \& Maraschi 1993). The spherical geometry corresponds to the model of Collin-Souffrin et al. (1996) where the hot phase constitutes a sphere at the center of the accretion flow surrounded by cold material possibly in the form of dense clouds or filaments. For simplicity, 


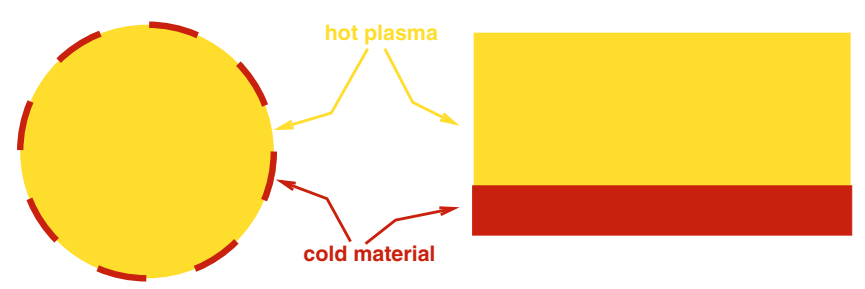

Fig. 1. The infinite slab accretion disc corona and spherical cloud geometry. The hot slab is divided into 10 horizontal layers with homogeneous temperatures. The sphere is divided into 5 concentric shells.

we assume that the material is homogeneously distributed at the sphere. For both models, the hot plasma is assumed to have an homogeneous density. It is however divided into a number of zones to account for temperature gradients that are computed according to the local energy balance.

Our model is then fully determined when the following parameters are given:

- Thomson optical depth $\tau_{\mathrm{T}}$ of the hot phase, defined along the radius of the sphere or the height of the hot slab.

- Density of the cold (homogeneous) material $n\left(\mathrm{~cm}^{-3}\right)$.

- Hydrogen column density of the cold material $N_{\mathrm{H}}\left(\mathrm{cm}^{-2}\right)$, or equivalently its Thomson optical depth $\tau_{\mathrm{c}}$.

- Ionization parameter of the cold material $\xi$, defined as $\xi=4 \pi F_{\text {bol }} / n$, where $F_{\text {bol }}$ is the integrated flux incident on the cold material surface. In the following $\xi$ is expressed in erg s${ }^{-1} \mathrm{~cm}$.

- Covering factor $C$ of the cold material, in the case of the spherical geometry. $C$ is the surface ratio covered by the cold clouds to that of the sphere. For a photon escaping from the hot sphere, $C$ represents the probability of entering into the cold medium.

\subsection{Numerical method}

We compute the escaping spectrum as follows. First, we use the non-linear Monte-Carlo code in order to get a first estimate of the high energy spectrum. When a LP photon escapes the hot plasma and enters the cold material, its energy is then reemitted at its surface in the form of reprocessed/reflected LP photons. Their energy and direction are drawn from the assumed angle dependent spectrum of the cold material. Note that the reprocessed photons may be either directed toward the hot plasma (i.e. reflected) or directed outward and escape the system (i.e. transmitted through the cold phase).

In the spherical model, we use a statistical method to model the homogeneous distribution of cold material around the sphere. When a photon LP escapes the hot sphere, a random number $\eta$ is drawn:

- if $\eta>C$, the LP photon truly escapes and its energy is used to build up the escaping spectrum;

- if $\eta<C$, the LP photon enters the cold material and its energy is either reflected toward the sphere or transmitted as described above.
At this stage the cold medium spectrum is arbitrary (although it is better if it is similar to a real reprocessed spectrum). We then inject the resulting equilibrium hot-phase spectrum as input in the TITAN/NOAR codes. This provides an estimate of the ionization and temperature structure of the cold phase as well as the reprocessed spectrum. Then we use the TITAN/ NOAR spectrum as the local spectrum of the cold material in the NLMC code to get a better estimate of the hot-phase spectrum, and so on. In general, convergence is achieved after 3-4 iterations.

\section{Results}

\subsection{Spherical model}

Figure 2 shows the resulting spectra for $C=0.3$ and $C=0.5$ and two values of the ionization parameter $\xi=300$ and $\xi=$ 3000 assuming a constant density in the cold phase.

For comparison we also show spectra that were obtained with the usual treatment of reprocessing (i.e. blackbody spectrum + neutral reflection). If all the radiation impinging on the cold material is absorbed and reemited as a blackbody, its temperature is related to the incident flux through the StefanBoltzmann law. Therefore the temperature $k T_{r}$ of the cold phase is related to the ionization parameter and density of the cold phase as follows:

$T_{r}=\left(\frac{\xi n}{4 \pi \sigma}\right)^{1 / 4}$

where $\sigma$ is the Stefan's constant. As a result, this equivalent blackbody temperature is not very sensitive to the incident flux. For our assumed density, $k T_{r}$ is 7 and $12 \mathrm{eV}$ for $\xi=300$ and 3000 respectively. In accordance, in the blackbody spectrum + neutral reflection simulations, the blackbody temperature was fixed at $k T_{r}=10 \mathrm{eV}$ in both cases, all the other relevant parameters being kept at the same values. Indeed, one can see from Fig. 2 that the blackbody spectrum peaks at nearly the same energy as the TITAN/NOAR spectrum.

Globally, for larger $C$, the X-ray spectra are always softer, i.e. the photon index of the primary emission $\Gamma$ (estimated with a least square fit of the $2-10 \mathrm{keV}$ primary spectrum) is larger. This is due to the increase of reprocessed cooling flux from the cold material at larger $C$ which leads to a lower temperature in the hot-phase.

There are however noticeable differences between spectra obtained with the usual treatment (i.e blackbody spectrum + neutral reflection) and the detailed treatment. The shape of the reprocessed spectrum obviously differs. But the Comptonised emission differs as well. In particular, the spectrum is much harder in the calculations using TITAN/NOAR. Indeed the reprocessed spectrum then is much broader than a simple blackbody, more photons being reprocessed at higher energy. This affects the Compton cooling in the hot phase (which is lower) together with the shape of the Comptonised spectrum which is harder also due to the relatively high energy of the seed photons. This effect is then amplified by the fact that the hard X-ray albedo is larger for a harder spectrum thus enhancing the fraction of the reflected/reprocessed photons at high energy. We 


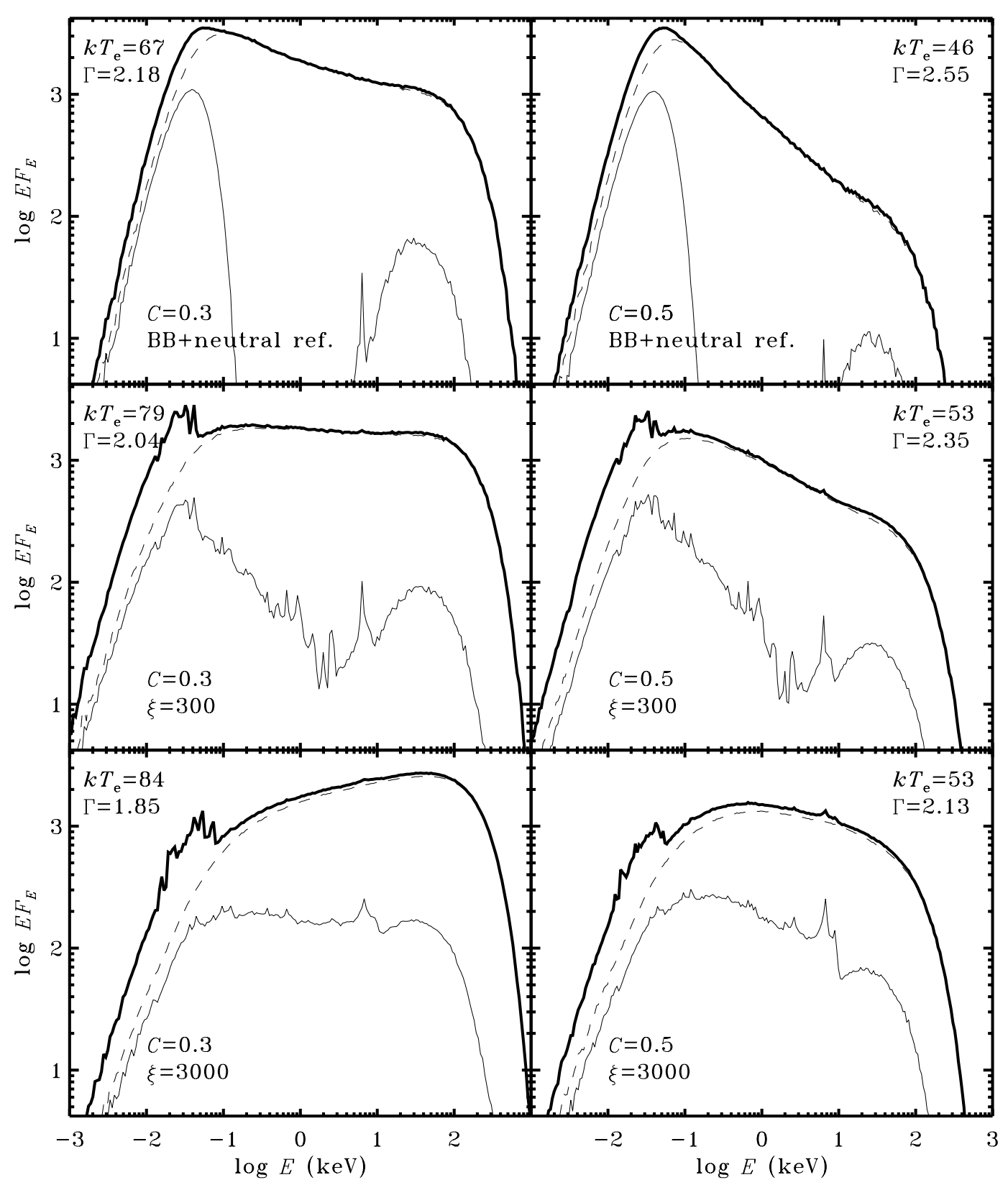

Fig. 2. Angle averaged escaping spectra for covering fractions $C=0.3$ (left-hand side) and $C=0.5$ (right-hand side). Thin curves: reprocessed/reflected spectrum. Dashes: comptonised spectrum. Thick curves: total observed spectrum (including the transmitted component). The upper panels show the results from NLMC calculations with the cold phase emission approximated by a blackbody + neutral reflection. The blackbody temperature is $k T_{r}=10 \mathrm{eV}$. The other panels are the results from the TITAN/NOAR/NLMC calculations for the following parameters of the cold medium: $n=3 \times 10^{14} \mathrm{~cm}^{-3}, N_{\mathrm{H}}=3 \times 10^{25} \mathrm{~cm}^{-2}\left(\tau_{\mathrm{c}}=24\right)$. In all of the simulations the Thomson optical depth of the hot plasma was fixed to $\tau_{\mathrm{T}}=1$. The assumed ionization parameter $\xi$ is indicated in each panel together with the resulting $2-10 \mathrm{keV}$ photon index of the comptonised emission $\Gamma$ and the volume averaged temperature of the hot phase $k T_{\mathrm{e}}$ (in $\mathrm{keV}$ ).

note that in the "blackbody" simulations, the total flux impinging on the cold material is reemitted toward the hot sphere (i.e. transmission through the cold medium is neglected). In contrast, in the TITAN/NOAR simulations, the transmitted flux ${ }^{1}$ represents about $15 \%$ of the impinging luminosity, despite our

\footnotetext{
${ }^{1}$ By "transmission" or "transmitted flux" we refer to the net energy flux across the cloud. This includes the radiation crossing the cloud without interaction, but also the reprocessed radiation that is outwardly reemitted. The latter is largely dominant.
}

choice of a relatively large hydrogen column density of the cold medium (corresponding to a Thomson optical depth $\tau_{\mathrm{c}}=24$ ).

This modest "loss" of soft photons leads to an increase of the temperature of a few percent and a hardening of the spectrum by $\Delta \Gamma \simeq 0.03$. Therefore, this effect does not contribute much to the resulting higher temperatures and harder spectra in the TITAN/NOAR case.

The hardening of the spectrum increases with $\xi$ (compare the spectra for $\xi=3000$ and $\xi=300$ ). The strongly ionised material has indeed a larger X-ray albedo, and more photons 


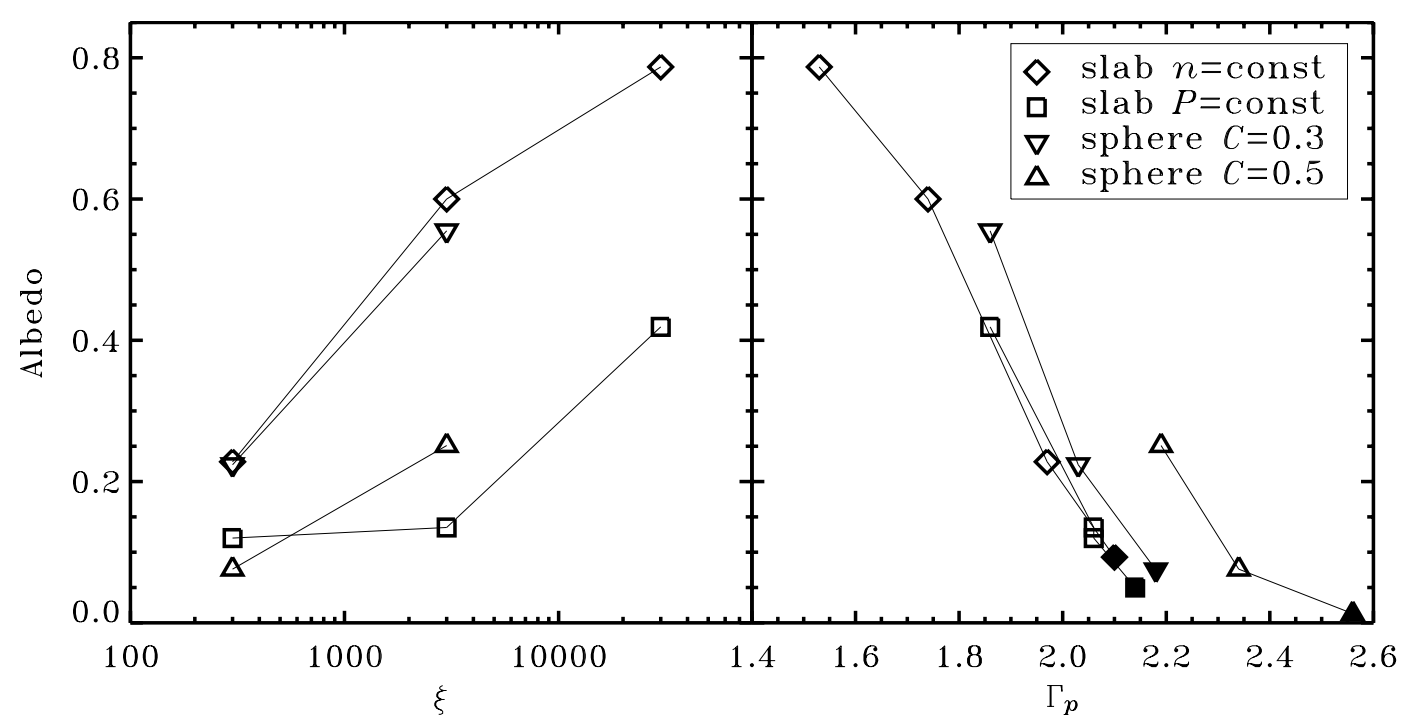

Fig. 3. X-ray albedo (i.e. fraction of the reflected/reprocessed luminosity emitted above $1 \mathrm{keV}$ ) as a function of the ionization parameter $\xi$ (left) and spectral index, $\Gamma_{\mathrm{p}}($ right), of the simulated spectra obtained from fits with the PEXRAV model (see text and Table 1). The results are plotted using a different symbol for each model, as shown on the figure. Filled symbols correspond to the blackbody and neutral reflection models.

Table 1. Spectral parameters obtained when fitting our simulated spectra in the $2-30 \mathrm{keV}$ range under XSPEC with the PEXRAV model plus a Gaussian line. $\Gamma_{\mathrm{p}}$ and $R$ are the PEXRAV photon index and reflection amplitude. $E_{\alpha}$ is the line centroid energy in $\mathrm{keV}$, and $E W$ is its equivalent width in $\mathrm{eV}$. During the fit the high energy cut-off energy $E_{\mathrm{c}}$ and the inclination angle were fixed at $400 \mathrm{keV}$ and 30 degrees respectively, and the abundances were fixed to standard.

\begin{tabular}{llcccc}
\hline \hline simulation & & $\Gamma_{\mathrm{p}}$ & $R$ & $E_{\alpha}$ & $E W$ \\
\hline$C=0.3$ & $T_{r}=10 \mathrm{eV}$ & 2.18 & 0.21 & 6.40 & 3.12 \\
$C=0.3$ & $\xi=300$ & 2.03 & 0.12 & 6.32 & 29.8 \\
$C=0.3$ & $\xi=3000$ & 1.86 & 0.01 & 6.61 & 126 \\
$C=0.5$ & $T_{r}=10 \mathrm{eV}$ & 2.56 & 0.36 & 6.40 & 8.66 \\
$C=0.5$ & $\xi=300$ & 2.34 & 0.21 & 6.31 & 35.6 \\
$C=0.5$ & $\xi=3000$ & 2.19 & 0.00 & 6.41 & 451 \\
\hline Slab & $T_{r}=10 \mathrm{eV}$ & 2.10 & 0.18 & 6.40 & 8.0 \\
Slab $n=$ const. & $\xi=300$ & 1.97 & 0.10 & 6.56 & 53.5 \\
Slab $n=$ const. & $\xi=3000$ & 1.74 & $9.5 \times 10^{-2}$ & 6.1 & 307 \\
Slab $n=$ const. & $\xi=30000$ & 1.53 & $2.1 \times 10^{-4}$ & 1.32 & 32 \\
Slab & $T_{r}=1 \mathrm{eV}$ & 2.14 & 0.13 & 6.40 & 9.53 \\
Slab $P=$ const. & $\xi=300$ & 2.06 & 0.16 & 6.4 & 12.9 \\
Slab $P=$ const. & $\xi=3000$ & 2.06 & 0.11 & 6.4 & 18.8 \\
Slab $P=$ const. & $\xi=30000$ & 1.86 & $8.7 \times 10^{-2}$ & 6.4 & 7.45 \\
\hline
\end{tabular}

are reprocessed at high energies. We note that the differences between the blackbody + neutral reflection model and the realistic one appear to be important even at moderate $\xi \sim 300$. The left panel of Fig. 3 shows the dependence of the X-ray albedo (defined as the fraction of the reflected/reprocessed luminosity emitted above $1 \mathrm{keV}$ ) on $\xi$. As the X-ray albedo also strongly depends on the shape of the incident spectrum (see Malzac et al. 2001), for a given $\xi$ the albedo can vary significantly with the geometry considered. The models with $C=0.3$ and $C=0.5$ have albedos differing by more than a factor of 2 , that is as important as the change in albedo when $\xi$ is increased by a factor of 10 . The right panel of Fig. 3 shows the resulting relation between albedo and spectral index. It is interesting to note that the difference of the albedos for the neutral and the low ionisation models $(\xi=300)$ is too low to account for the important changes in $\Gamma$ that we obtain. Indeed, the albedo changes by about 0.1 , implying an increase in the soft cooling flux of about $10 \%$, which, as noted above, is not sufficient to increase $\Gamma$ by $\Delta \Gamma \simeq 0.2$. This shows that the important difference in spectral slope between neutral and weakly ionised cases is essentially due to the different shape of the reprocessed spectrum below $1 \mathrm{keV}$ (as explained above) and not much to changes in the X-ray albedo.

Besides the spectral index, there are other parameters, such as the amplitude of the reflection features, that may be relevant to the observations. To derive those parameters from the simulated spectra in a way similar to those from observed spectra, we fitted our simulated spectra using the X-ray spectral fitting package XSPEC (Arnaud 1996). The fitting model was an efolded power law plus neutral reflection (PEXRAV, Magdziarz $\&$ Zdziarski 1995) and a Gaussian to model the iron line. The results are summarised in Table 1 and Fig. 4. In all cases we found that the spectral index obtained with PEXRAV $\Gamma_{\mathrm{p}}$, is very close to that derived through the $2-10 \mathrm{keV}$ least square fit. $\Gamma$. They show that the reflection amplitude $R$, measured with PEXRAV, strongly depends on the ionization parameter. It decreases by almost a factor of two between the neutral and $\xi=300$ case and for the highest ionization parameter $R$ is almost 0 . This is a well known effect due to the fact that the more ionised the reflector is, the more the reflected X-ray spectrum is similar to the incident one due to the predominance of Compton reflection over photoabsorption. As a consequence, the reflection bump appears relatively weaker in the overall spectrum. A further reduction of $R$ is due the destruction of the reflection component by Comptonisation in the hot phase (Malzac et al. 2001; Petrucci et al. 2001) depending on the Thomson optical depth of the hot sphere. 

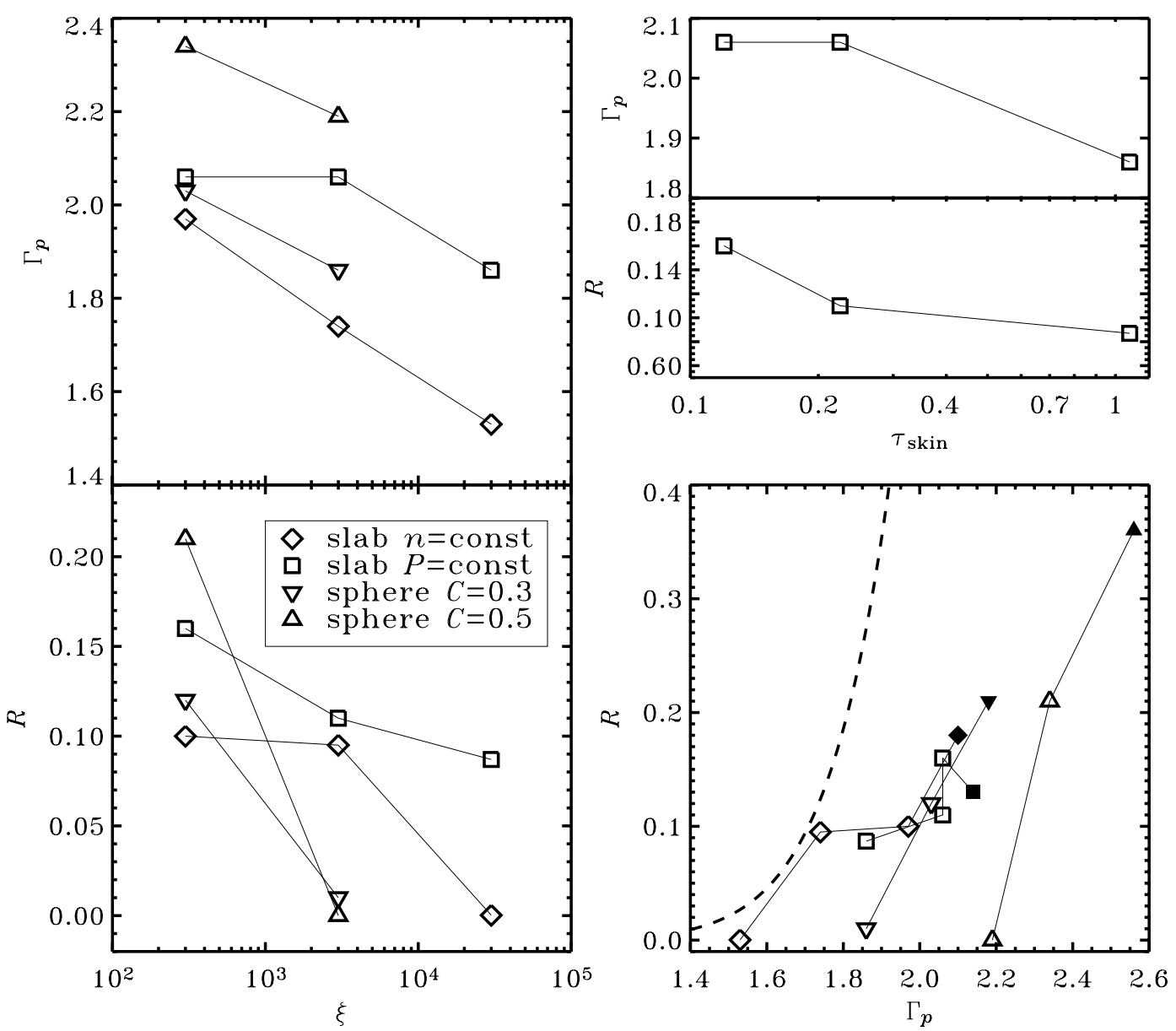

Fig. 4. Reflection amplitude $R$ and spectral index $\Gamma_{\mathrm{p}}$ of the simulated spectra obtained from fits with the PEXRAV model (see text and Table 1). The results are plotted using a different symbol for each model, as shown in the figure. Filled symbols correspond to the blackbody and neutral reflection models. Left panels: $R$ and $\Gamma$ as a function of $\xi$. Upper right panel: in the constant pressure case, $R$ and $\Gamma_{\mathrm{p}}$ as a function of the hot skin Thomson optical depth $\tau_{\text {skin. }}$ Bottom right panel: $R$ versus $\Gamma_{\mathrm{p}}$, the dashed curve shows the best fit power law approximation to the observed $R-\Gamma$ correlation in AGN as given by Zdziarski et al. (1999).

Contrary to the reflection component, the equivalent width of the iron line increases with the ionization parameter. Indeed in our simulations the line intensity increases with $\xi$ (by a factor of 10 between $\xi=300$ and $\xi=3000$ ), This is in agreement with previous studies showing that the line intensity increases between these two values of $\xi$ we considered (Matt et al. 1993, 1996). We note that in our fits the line energy is very weakly constrained. This is due to the low resolution of the fitted simulated spectra and also probably to the inadequacy of the Gaussian approximation to represent the much more complex profile of the actual line. This may explain the few unphysical red-shifted lines we obtain. Indeed, if estimated directly from the simulation, the line peak is always above $6.4 \mathrm{keV}$ and blue shifted at large $\xi$.

How do these results compare with the observations? The photon indices $\Gamma_{\mathrm{p}}>2$ obtained with PEXRAV for most of our simulations correspond to the softest observed sources (see e.g. Zdziarski et al. 2003). In the context of the spherical model this suggests that either the cold material is usually strongly ionised, or that in most sources, the covering fraction is lower than 0.3. On the other hand, the resulting $R$ is rather low compared to the average $R \sim 0.7$ in Seyfert galaxies, suggesting on the contrary that in most sources the covering fraction is larger. These problems can be easily overcomed by considering a slightly different geometry where the cold material, instead of being at the sphere surface would be at some distance. This would decrease both the cooling of the hot phase and the fraction of reflected photons that are Comptonised. Then, as shown by Malzac (2001), for the same covering fraction the spectrum is harder and the reflection component is stronger. We also note that, in Seyfert galaxies, a distant molecular torus could provide a strong additional contribution to the observed reflection component (Malzac \& Petrucci 2002).

\subsection{Slab corona model}

In the spherical model, changes in the covering fraction of cold material may produce a range of $\mathrm{X}$-ray photon indices that includes the observed range. On the other hand the slab corona model, in its simplest version, does not allow such freedom; the photon index is tightly constrained (Haardt \& Maraschi 1993). Detailed simulations using the blackbody + neutral reflection approximation (Stern et al. 1995b) have shown that the range of $\Gamma$ expected in this geometry tends to be larger than $\sim 2$, 


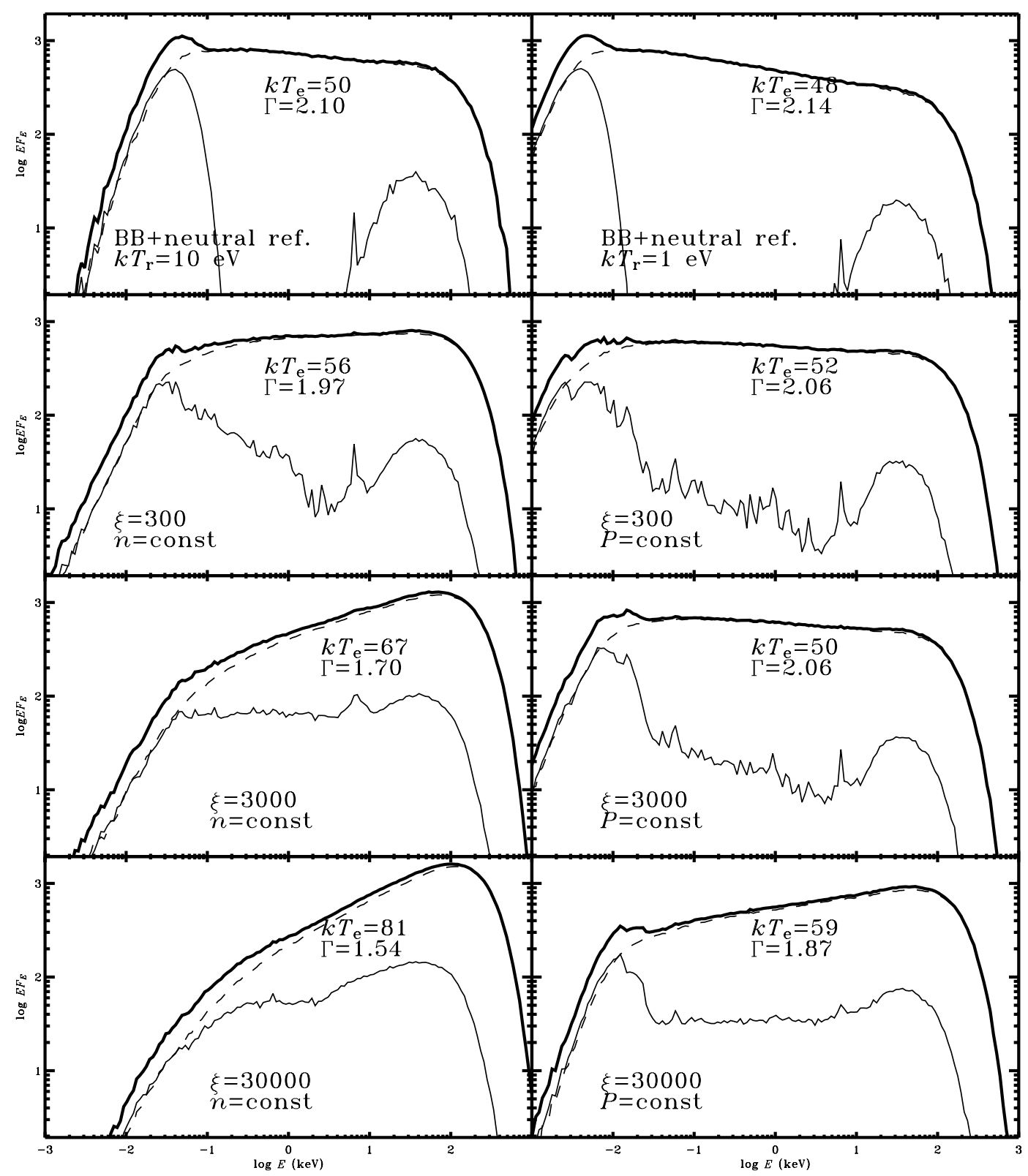

Fig. 5. Angle averaged escaping spectra for a plane-parallel geometry. The bottom panels are the results from the TITAN/NOAR/NLMC calculations for the same parameters of the cold medium as in Fig. 2, and assuming a constant density (left hand side) and a constant pressure (right hand side) in the cold phase. The upper panels show the results from NLMC calculations with the cold phase emission approximated by a blackbody + neutral reflection for two different blackbody temperatures $k T_{r}=10 \mathrm{eV}$ (left hand side) $k T_{r}=1 \mathrm{eV}$ ( right hand side). Thin curves: reprocessed/reflected spectrum. Dashes: comptonised spectrum. Thick curves: total observed spectrum. In all panels, the Thomson optical depth of the hot plasma is $\tau_{\mathrm{T}}=1$. The assumed ionization parameter $\xi$ is indicated in each panel together with the resulting $2-10 \mathrm{keV}$ photon index of the Comptonised emission $\Gamma$ and the volume averaged temperature of the hot phase $k T_{\mathrm{e}}$ (in $\mathrm{keV}$ ).

while the average $\Gamma$ in Seyfert 1 galaxies is $\sim 1.8$. Observed spectra of Seyfert galaxies and galactic black hole sources can be much harder with photon indices as low as $\Gamma \sim 1.4$. Overcoming this problem requires some complications, such as considering the effects of an out-flowing corona (Beloborodov 1999; Malzac et al. 2001) or the presence of holes in the disc (Zdziarski et al. 1998). As a consequence, the simple slab coronal model considered here was often considered as ruled out on this basis.

As our more realistic treatment of reprocessing and ionization tends to produce harder spectra, it is interesting to see whether this would be sufficient to reconcile the model with the observations. The left panels of Fig. 5 compare spectra for a slab geometry and both the blackbody approximation and realistic reprocessing for $\xi=3 \times 10^{2}, \xi=3 \times 10^{3}$ and $\xi=3 \times 10^{4}$, assuming a constant density in the cold phase. Unlike those of the spherical model, these slab spectra are angle dependent. However the weak angular dependence obtained in our detailed reprocessing simulations does not differ qualitatively from what is found in the standard "blackbody" + neutral reflection treatment that was already studied in several papers (see e.g. Malzac et al. 2001). For the sake of simplicity, 
we will consider the properties of the angle-averaged spectra only, since the aim of this work is to evaluate the main effects of a detailed treatment of reprocessing and ionization.

The spectral indices from the $2-10 \mathrm{keV}$ least square fit are $\Gamma=2.10$ for the "blackbody" simulation and $\Gamma=1.97,1.70$ and 1.54 respectively for the ionised cases. Thus although the hardening of the spectrum is significant, the model will be able to reproduce the spectra of the hardest sources only if the disc is strongly ionised.

Since, in our calculation, we neglect the contribution of internal viscous dissipation to the disc emission, our spectra are the hardest possible for the slab corona geometry. Any intrinsic disc emission would make the spectra softer and strengthen the requirements for extreme ionization.

In the TITAN/NOAR simulations shown in Fig. 2 and the left panels of Fig. 5 we assumed a constant density in the cold material. This assumption might be a reasonable approximation in the case of the small-scale magnetically confined clouds of the model of Collin-Souffrin et al. (1996). In the case of the accretion disc corona however, there are certainly strong density gradients driven by the pressure equilibium. In general, due to the ionization thermal instability, it breaks into two well defined layers: a low density skin at Compton temperature that is almost completely ionised, atop the cooler, high density, low ionization material of the internal disc. The sharp transition between the two layers contrasts with the smooth ionization gradients obtained in the constant density case. This characteristics was studied in a number of papers (Raymond 1993; Ko \& Kallman 1994; Różańska \& Czerny 1996; Nayakshin et al. 2000; Dumont et al. 2002) that showed it has strong impact on the reprocessed spectrum. The reprocessed spectrum can then be described as formed by two components associated with two layers. The hot skin component is essentially dominated by Compton reflection with a spectral shape almost indistinguishable from the irradiating spectrum, while the deeper component corresponds to almost neutral reprocessing (Done \& Nayakshin 2001; Ballantyne et al. 2001).

Our results indicate that the primary spectrum is sensitive to the shape of the reprocessed spectrum. Therefore, one may wonder what effect different assumptions made about the structure of the cold material (constant density, constant pressure or hydrostatic equilibrium) may have on the shape of the overall equilibrium spectral energy distribution.

In the right panels of Fig. 5, we show the resulting spectra for the slab geometry and a constant pressure in the cold phase. For the 3 simulations with constant pressure the resulting Thomson depth of the ionised skin $\tau_{\text {skin }}$ is $0.120,0.225$, and 1.08 respectively for $\xi=3 \times 10^{2}, 3 \times 10^{3}$, and $3 \times 10^{4}$. As compared to the constant density case, and in agreement with previous studies, the reprocessed spectrum is much softer in the X-ray range due to the strong fraction of photons that are Compton reflected in the hot skin and the low ionization state of the deeper layers. For the same reasons, the UV spectrum formed in the colder deeper regions is sharper and peaks at lower frequency. The soft seed photons are thus softer on average and as a consequence the primary X-ray spectrum is slightly steeper than its constant density counterpart.
Despite the reprocessed spectrum, in its lowest energy part, is now closer to a blackbody spectrum, the primary X-ray emission still differs from that obtained in the blackbody approximation.

To facilitate the comparison, the "blackbody" case shown in upper right panel of Fig. 5 is for $k T_{r}=1 \mathrm{eV}$, so that the blackbody peaks nearly at the same energy as the realistic reprocessed UV spectrum. For both $k T_{r}=1 \mathrm{eV}$ and $k T_{r}=10 \mathrm{eV}$ the primary X-ray spectrum is steeper than in the TITAN/NOAR simulation.

The results of the XSPEC fitting procedure are shown in Table 1 . For a very thick reflector subtending a solid angle $2 \pi$, we expect $R=1$. On the other hand, the reflection coefficient we derive are relatively low, $\sim 0.1-0.2$ even in the case of neutral reflection. This is a known effect resulting from the destruction of the reflection component by Comptonisation in the hot phase (Malzac et al. 2001; Petrucci et al. 2001). In general, this effect is important for all geometries where the reflector is seen through the hot plasma, such as the slab or spherical geometry considered in Sect. 3.1.

Contrary to the case of a constant density, in the constant pressure models, the spectral index is quite insensitive to the ionization parameter. The spectrum hardens by less than $\Delta \Gamma \simeq$ 0.2 when $\xi$ is increased by 2 orders of magnitude. In this case the ionization effects are not sufficient, to reconcile the slab corona model with the observations. This latter result confirms a similar conclusion reached by Nayakshin \& Dove (2001), on the basis of a simplified treatment of the hot skin model. On the other hand, if the reflector has a constant density, we find that the different ionization parameters could explain the whole range of observed spectral indices. This however would require a very wide range of ionization parameters, with an extremely ionised reflector in the hardest sources. Moreover, although the constant density is plausible in the context of the cloud model of Sect. 3.1, an accretion disc with a constant density seems quite unrealistic.

Finally, both for constant density and constant pressure a detailed treatment of reprocessing appears to be important. The usual blackbody + neutral reflection approximation appears inappropriate for an accurate determination of the equilibrium spectra, even at low ionization.

\section{Conclusions}

We calculated the equilibrium spectra in the context of the twophase models for the emission from Seyfert galaxies and black hole binaries. For the first time, we included a detailed treatment of reprocessing and ionization and thermal balance in the cold phase.

The resulting broad band spectrum differs significantly from what obtained using the usual "blackbody + neutral reflection" approximation. In particular, our realistic treatment of reprocessing leads to higher hot-phase temperatures associated with harder X-ray spectra. Surprisingly the effect is strong even at low ionization parameters. This is because even if the X-ray albedo is not significantly affected by the weak ionizing illumination, the shape of the reprocessed spectrum below $1 \mathrm{keV}$ is much broader than a simple blackbody. 
For the slab model, we performed simulations with two different prescriptions for the cold phase, namely a constant density and a constant pressure. The comparisons between the two cases indicate that the overall spectral shape is also quite sensitive to the physical structure of the cold phase. A slab model at constant density can account for the hardest observed spectra for large values of the ionization parameters. In the case of constant pressure disc however, the hardening is not strong enough to reconcile the model with the even harder spectra observed in Seyfert galaxies and Galactic black holes.

The equilibrium spectra of a two-phase system thus appear to be significantly affected by the shape of the reprocessed spectrum, even at low ionization parameters. A detailed treatment of reprocessing appears to be required for most observationally relevant cases.

Acknowledgements. This work was partly supported by the European Commission (contract number ERBFMRX-CT98-0195, TMR network "Accretion onto black holes, compact stars and protostars"). J.M. also acknowledges fundings from the MURST (COFIN98-02-15-41) and PPARC. J.M. acknowledges a travel grant from the GDR PCHE. We are grateful to Suzy Collin and Pierre-Olivier Petrucci for enlightening discussions.

\section{References}

Abrassart, A. 2000, Adv. Sp. Res., 25, 465

Arnaud, K. A. 1996, Astronomical Data Analysis Software and Systems V, ed. Jacoby G., \& Barnes J., ASP Conf. Ser., 101, 17

Ballantyne, D. R., Ross, R. R., \& Fabian, A. C. 2001, MNRAS, 327, 10

Balucinska-Church, M., Belloni, T., Church, M. J., \& Hasinger, G. 1995, A\&A, 302, L5

Beloborodov, A. M. 1999, ApJ, 510, L123

Collin-Souffrin, S., Czerny, B., Dumont, A.-M., \& Życki, P. 1996, A\&A, 314, 393

Done, C., \& Nayakshin, S. 2001, MNRAS. 328, 616

Dumont, A.-M., Abrassart, A., \& Collin, S. 2000, A\&A, 357, 823

Dumont, A.-M., Czerny, B., Collin, S., \& Zycki, P. T. 2002, A\&A, 387,63

George, I. M, \& Fabian, A. C. 1991, MNRAS, 249, 352
Haardt, F., \& Maraschi, L. 1991, ApJ, 380, L51

Haardt, F., \& Maraschi, L. 1993, ApJ, 413, 507

Ko, Y.-K., \& Kallman, T. R. 1994, ApJ, 431, 273

Magdziarz, P., \& Zdziarski, A. A. 1995, MNRAS, 273, 837

Malzac, J. 2001, MNRAS, 325, 1625

Malzac, J., \& Jourdain, E. 2000, A\&A, 359, 843

Malzac, J., \& Celotti, A. 2002, MNRAS, 335, 23

Malzac, J., \& Petrucci, P. O. 2002, MNRAS, 336, 1209

Malzac, J., Beloborodov, A. M., \& Poutanen J. 2001, MNRAS, 326, 417

Matt, G., Fabian, A. C., \& Ross, R. R. 1993, MNRAS, 262, 179

Matt, G., Fabian, A. C., \& Ross, R. R. 1996, MNRAS, 278, 1111

Nandra, K., \& Pounds, K. A. 1994, MNRAS, 268, 405

Nayakshin, S., \& Dove, J. B. 2001, ApJ, 560, 885

Nayakshin, S., \& Kallman, T. R. 2001, ApJ, 546, 406

Nayakshin, S., Kazanas, D., \& Kallman T. R. 2000, ApJ, 537, 833

Petrucci, P. O., Merloni, A., Fabian, A., Haardt, F., \& Gallo, E. 2001, MNRAS, 328, 501

Poutanen, J., \& Svensson, R. 1996, ApJ, 470, 249

Raymond, J. C. 1993, ApJ, 412, 267

Rees, M. J. 1987, MNRAS, 228, 47

Ross, R. R., \& Fabian, A. C. 1993, MNRAS, 261, 74

Różańska, A., \& Czerny, B. 1996, AcA, 46, 233

Różańska, A., \& Czerny, B. 2000, A\&A, 360, 1170

Shapiro, S. L., \& Lightman, A. P. 1976, ApJ, 204, 555

Sincell, M. W., \& Krolik, J. H. 1997, ApJ, 476, 605

Stern, B. E., Begelman, M. C., Sikora, M., \& Svensson, R. 1995a, MNRAS, 272, 291

Stern, B. E., Poutanen, J., Svensson, R., Sikora, M., \& Begelman, M. C. $1995 b$, ApJ, 449, L13

Svensson, R., \& Zdziarski, A. A. 1994, ApJ, 436, 599

Shakura, N. I., \& Sunyaev, R. A. 1973, A\&A, 24, 337

Sunyaev, R. A., \& Titarchuk, L. G. 1980, A\&A, 86, 121

Walter, R., \& Fink, H. H. 1993, A\&A, 274, 105

Zdziarski, A. A., Poutanen, J., Mikolajewska, J., et al. 1998, MNRAS, 301,435

Zdziarski, A. A., Lubiński, P., \& Smith, D. A. 1999, MNRAS, 303, L11

Zdziarski, A. A., Lubiński, P., Gilfanov, M., \& Revnivtsev, M. 2003, MNRAS, 342, 355

Życki, P. T., Collin-Souffrin, S., \& Czerny, B. 1995, MNRAS, 277, 70

Życki, P. T., Krolik, J. H., Zdziarski, A. A., \& Kallman, T. R. 1994, ApJ, 437, 597 